\title{
Hemin-activated macrophages home to the pancreas and protect from acute pancreatitis via heme oxygenase-1 induction
}

\author{
Ikuo Nakamichi,,1,2 Aida Habtezion,, 1,2 Bihui Zhong,,1,2 Christopher H. Contag, ${ }^{3}$ \\ Eugene C. Butcher, ${ }^{2,4}$ and M. Bishr Omary ${ }^{1,2}$ \begin{abstract}
${ }^{3}$ Molecular Imaging Program and Department of Pediatrics, Stanford University, Stanford, California, USA. ${ }^{4}$ Department of Pathology,
\end{abstract} \\ 1Department of Medicine, VA Palo Alto Health Care System, Palo Alto, California, USA. ${ }^{2}$ Stanford University, Stanford, California, USA. \\ VA Palo Alto Health Care System, Palo Alto, California, USA.
}

\begin{abstract}
Hemin upregulates heme oxygenase-1 (HO-1), a stress-induced enzyme implicated in protection from a variety of injuries while its related isoform $\mathrm{HO}-2$ is constitutively expressed. The role of hemin or $\mathrm{HO}-1$ in the pancreas and their potential modulation of pancreatic injury are unknown. We show that HO-1 is induced in pancreatitis caused by caerulein and more prominently in severe pancreatitis caused by feeding a choline-deficient diet (CDD). Intraperitoneal hemin administration dramatically increases peritoneal and pancreas macrophages that overexpress $\mathrm{HO}-1$ in association with pancreatic induction of the chemoattractants monocyte chemotactic protein-1 and macrophage inflammatory protein-1 $\alpha$ but not RANTES or macrophage inflammatory protein-2. Hemin administration before CDD feeding protected 8 of 8 mice from lethality while 7 of 16 controls died. Protection is mediated by HO-1-overexpressing macrophages since hemin-primed macrophages home to the pancreas after transfer to naive mice and protect from CDD-induced pancreatitis. Suppression of hemin-primed peritoneal cell HO-1 using HO-1-specific small interfering RNA prior to cell transfer abolishes protection from CDD-induced pancreatitis. Similarly, hemin pretreatment in caerulein-induced pancreatitis reduces serum amylase and lipase, decreases pancreatic trypsin generation, and protects from lung injury. Therefore, hemin-like compounds or hemin-activated macrophages may offer novel therapeutic approaches for preventing acute pancreatitis and its pulmonary complication via upregulation of $\mathrm{HO}-1$.
\end{abstract}

\section{Introduction}

Hemin upregulates heme oxygenase-1 (HO-1), a stress-induced enzyme implicated in protection from a variety of injuries while its related isoform HO-2 is constitutively expressed (1-6). HOs catalyze the rate-limiting step in heme degradation to produce $\mathrm{CO}$, iron, and biliverdin in equimolar amounts $(4,6,7)$. Biliverdin is then reduced to bilirubin by biliverdin reductase. HO-1 $(32 \mathrm{kDa}$; also referred to as heat shock protein-32) and $\mathrm{HO}-2(36 \mathrm{kDa})$ are unique gene products that share nearly $40 \%$ identity at the amino acid level $(4,6)$. The role of HO-1 and hemin in the exocrine pancreas and their potential modulation of pancreatic injury are unknown. The only descriptions of HO- 1 in the exocrine pancreas are in two 1997 reports that demonstrate $\mathrm{HO}-1$ induction in caerulein-mediated mouse pancreatitis $(8,9)$ and in AR42J rat acinar cells exposed to $\mathrm{H}_{2} \mathrm{O}_{2}$ or $\mathrm{CdCl}_{2}$ (9). HO-1 null mice develop anemia and high tissue iron levels with consequent oxidative damage in the liver, glomerulonephritis, and splenomegaly. These mice have more than $80 \%$ embryolethality, and most surviving mice die within 6 months due to presumed multiorgan failure $(1,2,10)$. There is also a single reported case of HO-1 deficiency identified in a child who died at the age of 6 with renal disease and intracranial hemorrhage $(11,12)$. The effect of $\mathrm{HO}-1$ absence on pancreatic pathology in humans and mice is unknown.

Nonstandard abbreviations used: BUN, blood urea nitrogen; CDD, cholinedeficient diet; HO-1, heme oxygenase-1; MCP-1, monocyte chemotactic protein-1; MIP-1 $\alpha$, macrophage inflammatory protein-1 $\alpha$; MPO, myeloperoxidase; siRNA, small interfering RNA.

Conflict of interest: The authors have declared that no conflict of interest exists.

Citation for this article: J. Clin. Invest. 115:3007-3014 (2005).

doi:10.1172/JCI24912.
Hemin is the prosthetic moiety for a broad range of proteins that play essential roles in oxygen delivery, mitochondrial function, and signal transduction, including hemoglobin, cytochromes, prostaglandin endoperoxide, and nitrous oxide synthases, catalase, and peroxidases. Various formulations of hemin such as hematin and other alternate intravenous formulations (e.g., panhematin) are currently available and have been used in patients since the 1970 s with minimal side effects reported to successfully treat acute porphyrias, to control liver allograft failure due to recurrence of erythropoietic protoporphyria, and in patients with thalassemia intermedia (13-16). Hemin is also an established agent for HO-1 induction in several tested cultured cells and in vivo (9, 17-20).

Conversion of trypsinogen to active trypsin within pancreatic acinar cells is an important event in developing acute pancreatitis. Furthermore, it has been well demonstrated that infiltrating neutrophils significantly contribute to this intrapancreatic trypsin activation (21). However, the mechanisms and signals that mediate neutrophil or other inflammatory cell (such as macrophage) recruitment into the pancreas are less well understood. In this report we demonstrate a novel protective role for hemin in experimental mouse pancreatitis and provide a cellular mechanistic basis for such protection. This role is mediated by HO- 1 and leads to recruitment of HO-1 expressing macrophages to the pancreas.

\section{Results}

Hemin administration protects from injury in experimental acute pancreatitis. We examined HO-1 expression in 2 established mouse pancreatitis models: (a) caerulein hyperstimulation, which causes mild pancreatitis, and (b) feeding with a choline-deficient 

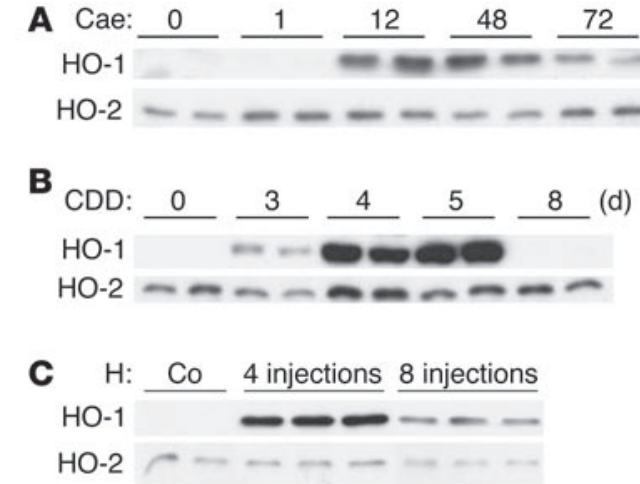

(h)
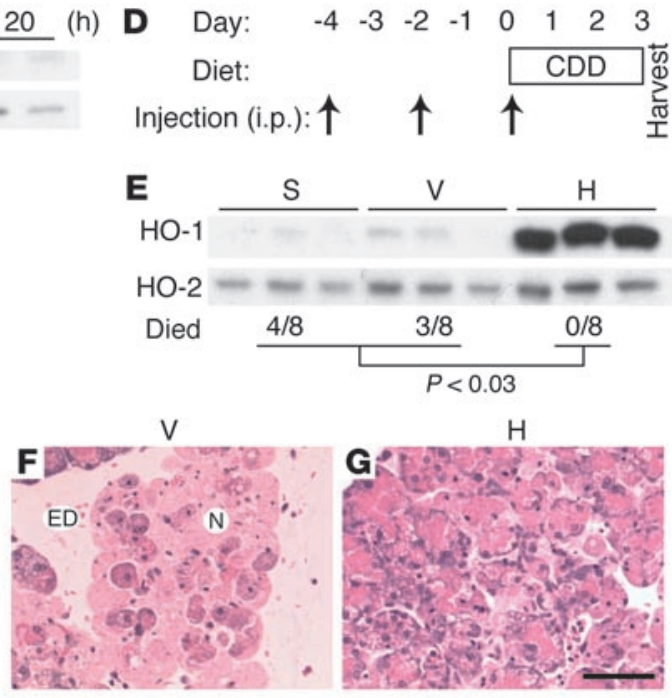

$\mathrm{H}$

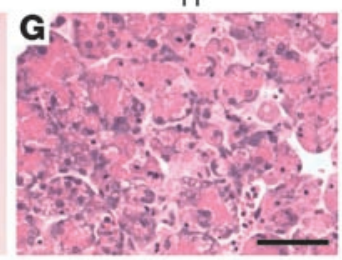

\section{Figure 1}

Effect of hemin or pancreatic injury on HO-1 induction and inhibition of pancreatic injury by hemin. (A and B) Total tissue homogenates were obtained from pancreata of caerulein-injected (cae-injected) or CDD-fed mice. Two age- and sex-matched mice were used for each time point. Homogenates were tested by blotting, using antibodies to HO-1 or HO-2. (C) Mice (3 mice/condition) were given hemin (H) by i.p. injection (4 times during 1 week or 8 times during 2 weeks). Two control (Co) mice were injected with vehicle alone. Pancreatic homogenates were then obtained and blotted with anti-HO-1 and anti-HO-2. (D-G) Mice were injected with saline (S), hemin, or vehicle (V) (8 mice/group) 3 times (arrows) followed by feeding with CDD for 3 days, then harvesting of the pancreata. HO-1 and HO-2 were analyzed by blotting of pancreatic homogenates ( 3 mice/group). The number of mice that died in each cohort of 8 mice is shown, and the survival difference was significant $(P<0.03)$ when comparing controls (saline and vehicle) with hemin-injected mice. Representative H\&E stainings of pancreata from mice that survived CDD feeding are shown. Scale bar: $50 \mu \mathrm{m}$. Note the marked pancreas edema (ED) and necrosis (N) in the saline- (not shown) and vehicle-injected mice as compared with the hemin group.

diet (CDD), which causes severe pancreatitis (22-24). HO-1 levels increased significantly (particularly in the more severe CDD model) and reversibly in the 2 tested pancreatitis models, while HO-2 levels were minimally altered (Figure 1, A and B). Given the high HO-1 induction we observed in the caerulein and CDD pancreatitis models, we hypothesized that HO-1 induction may be associated with modulation of the extent of pancreatic injury. We tested this hypothesis by first assessing the effect of administering hemin on pancreatic HO-1 levels. Hemin i.p. administration into mice dramatically induced pancreatic HO-1 but not $\mathrm{HO}-2$, though repeat injections blunted $\mathrm{HO}-1$ upregulation (Figure 1C). More importantly, hemin priming of mice prior to feeding with CDD (Figure 1D) provided complete protection from lethality as compared with vehicle- or saline-injected mice (Figure 1, E-G). This protection was associated with marked induction of $\mathrm{HO}-1$ in pancreas tissue homogenates (Figure 1E). The effect of hemin i.p. administration on HO-1 induction in the pancreas (Figure 1C) and isolated peritoneal cells depended on the tissue in that HO-1 levels in several other organs including the spleen and bone marrow were minimally altered (Supplemental Figure 1; supplemental material available online with this article; doi:10.1172/ JCI24912DS1). These results indicate that HO-1 is markedly induced during experimental acute pancreatitis and that hemin administration mimics and even accentuates the pancreatitisassociated marked HO-1 upregulation.

We also examined the potential protective effect of hemin in caerulein-induced pancreatitis (Figure 2). Although no significant difference was found in the overall histology score (see Methods) when comparing caerulein-treated mice given vehicle versus hemin (not shown), hemin priming prior to caerulein administra- tion significantly decreased serum amylase and lipase levels and pancreatic trypsinogen activation (Figure 2, B and C). Pancreatic myeloperoxidase (MPO) activity could not be assessed adequately since hemin plus saline (without caerulein) alone had elevated MPO levels (Figure 2C). Notably, hemin priming conferred a dramatic protection from the lung injury that associates with caerulein pancreatitis, as supported by histologic assessment (Figure 2, D-F). Despite baseline elevation of lung MPO activity in hemin plus saline-treated mice, the extent of lung injury after caerulein administration was able to unmask a significant difference in lung MPO activity after caerulein plus hemin administration (Figure 2D). Hence, hemin protected from pancreatitis-associated injury when tested in 2 independent pancreatitis models.

Peritoneal macrophages home to the pancreas after hemin administration. In order to understand the mechanism of protection by hemin, we asked which cells account for the induced HO-1 during pancreatitis. Previous studies showed that hemin induces HO-1 in a variety of epithelial and nonepithelial cell types $(9,17-20)$. HO- $1^{+}$cells that are induced in the pancreas after pancreatic injury are mesenchymal and not epithelial, as determined by HO-1 colocalization with vimentin but not with keratin-8 staining (Supplemental Figure 2, A-F). Interestingly, pancreatic $\mathrm{HO}-1^{+}$cells (after hemin administration) did not stain with the endothelial marker MJ7/18 (Supplemental Figure 2, G-I), but most of HO-1 colocalized primarily with F4/80 (>90\%) and to a much lesser extent with Gr-1 (Figure 3, A-D) that represent macrophage $\left(\mathrm{F} 4 / 80^{+}\right)$and neutrophil $\left(\mathrm{Gr}-1^{+}\right)$markers, respectively. Staining of Mac-1 (another macrophage marker) provided similar results to those obtained with F4/80 (not shown). This suggests that $\mathrm{HO}-1$ induction during pancreatic injury occurs preferentially in pancreatic macrophages. 

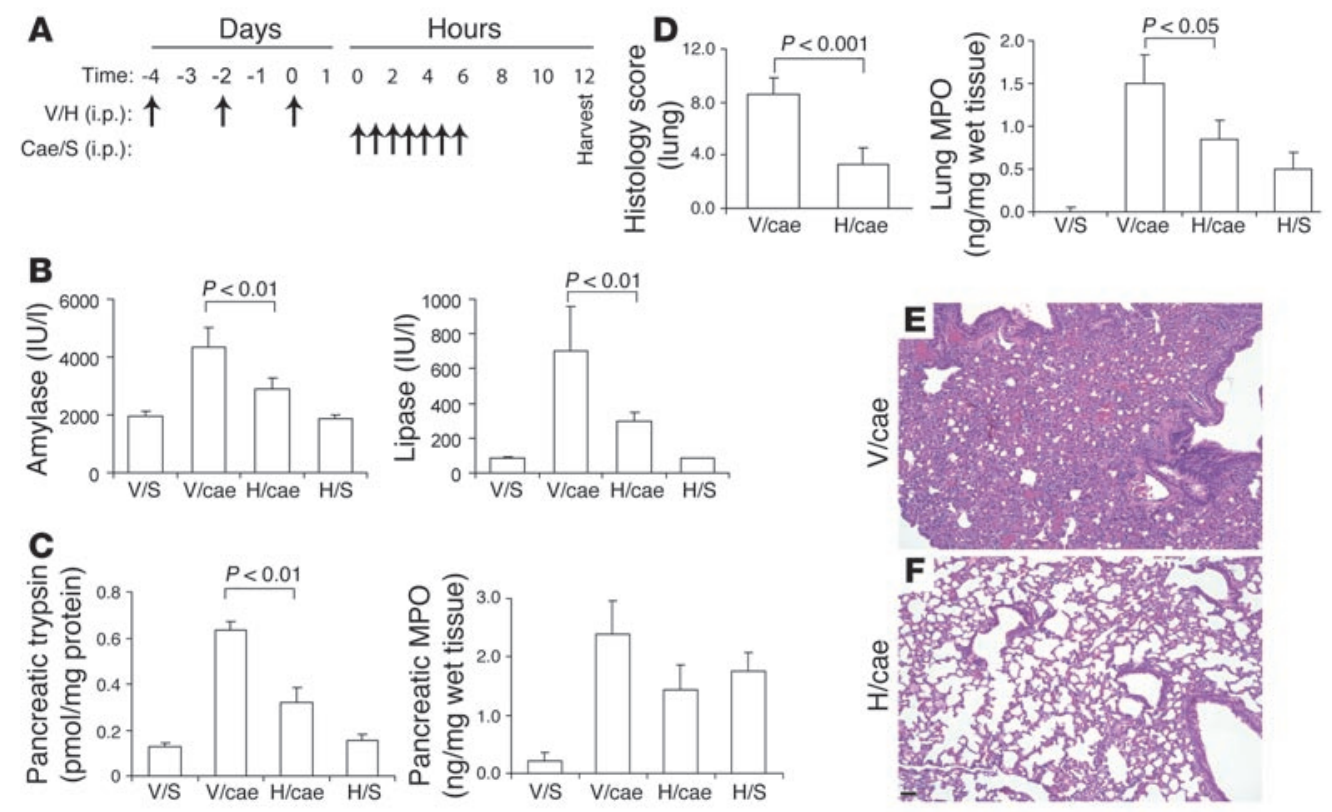

Figure 2

Effect of hemin on caerulein-induced pancreatitis and its pulmonary complications. (A and B) Mice were injected i.p. with hemin or vehicle (7 mice/group) every other day (3 times; arrows) followed by injection with either saline or caerulein hourly ( 7 times; arrows) and then harvesting of the pancreata and lungs at 12 hours after first saline or caerulein injection. Sera were collected for amylase/lipase measurement. (C-E) Pancreatic and lung tissues (from $\mathbf{A}$ and $\mathbf{B}$ ) were used to determine trypsin (pmol/mg protein) and MPO (ng/mg wet tissue) enzyme activities and to assess extent of tissue injury. Data are presented as mean \pm SD. A representative H\&E staining of lung is shown. Scale bar: $50 \mu m$. Note the marked hemorrhage and alveolar collapse in vehicle- $(\mathbf{E})$ compared with hemin- $(\mathbf{F})$ pretreated mice receiving caerulein.

Given the dramatic effect of HO-1 induction in the pancreas by simple i.p. injection of hemin in the absence of pancreatitis (Figure 1C) and the observed protection from pancreatitis by hemin (Figures 1, E-G, and 2), we quantified the effect of hemin on pancreatic macrophages in the presence or absence of CDD feeding. Comparison between peritoneal cells of hemin- and vehicle-injected mice showed a more than 2-fold increase in total peritoneal cells and a more than 4-fold increase in peritoneal macrophages (Supplemental Table 1). Quantification of the number of pancreatic macrophages showed a significant increase upon hemin administration with or without CDD feeding that paralleled the frequency of hemin injections (Figure 3E). Hemin treatment induced recruitment of $\mathrm{HO}-1^{+}$macrophages to the pancreas without causing pancreatitis (using histological and serologic criteria, not shown), which suggests that hemin is directly or indirectly involved in chemoattraction of macrophages to the pancreas. Earlier studies showed that hemin can induce the chemokine monocyte chemotactic protein-1 (MCP-1) in immortalized rat proximal tubular epithelial cells (18) and can result in leukocyte activation (20). Therefore we tested the hypothesis that hemin upregulates chemokines, particularly those that attract macrophages. Hemin or vehicle were injected i.p. into mice only once followed by harvesting of pancreata from injected or noninjected mice after 24 hours in order to measure the mRNA levels of several chemokines. As shown in Figure 3F, MCP-1 and macrophage inflammatory protein-1 $\alpha$ (MIP-1 $\alpha$ ) but not RANTES or MIP-2 levels increased dramatically after a single i.p. dose of hemin. Hence, the hemininduced increase in MCP-1/MIP-1 $\alpha$ and/or other chemoattractants provides a plausible explanation for the observed increase in pancreas $\mathrm{HO}-1^{+}$macrophages.
The increase in $\mathrm{HO}-1^{+}$peritoneal and pancreatic macrophages in response to hemin raised the hypothesis that hemin administration induces homing of peritoneal macrophages to the pancreas, which in turn are responsible for the observed protection afforded by hemin. We tested this hypothesis by carrying out the transfer experiment outlined in Figure 4A. Whole body in vivo imaging following transfer of $\mathrm{Mac}-1^{+}$peritoneal cells from hemin-pretreated luciferase-overexpressing mice showed that a significant number of the injected cells remained in the peritoneal cavity of the nonluciferase recipient mice given hemin while recipients given vehicle dissipated (Figure 4, B-D). Importantly, the double-positive luciferase $/ \mathrm{Mac}-1^{+}$macrophages in mice given hemin were specifically localized in the pancreas but not in the liver (Figure 4, B-D) or other abdominal organs (not shown). Presence of luciferase/Mac-1 ${ }^{+}$ cells within the pancreas was confirmed by staining (Figure 4, E-G), indicating that hemin-primed peritoneal macrophages are capable of migrating to the pancreas of hemin-treated but not vehicle-treated recipient mice.

Peritoneal cells protect from CDD-induced pancreatitis via HO-1 induction. Confirmation that peritoneal cells were indeed responsible for imparting the protective effect of CDD-induced pancreatic damage was carried out by transferring peritoneal cells obtained from hemin- or vehicle-treated mice into naive mice, followed by exposure of the recipient mice to CDD (Figure 5A). Significantly lower serum amylase and blood urea nitrogen (BUN) levels (elevated upon dehydration and renal injury) were noted in mice receiving hemin-activated cells (Figure 5B). Half of the mice receiving cells from vehicle-treated animals developed macroscopic hemorrhagic pancreatitis ( 3 of 6 mice) while none of the mice receiving heminactivated cells had any macroscopic evidence of hemorrhage 

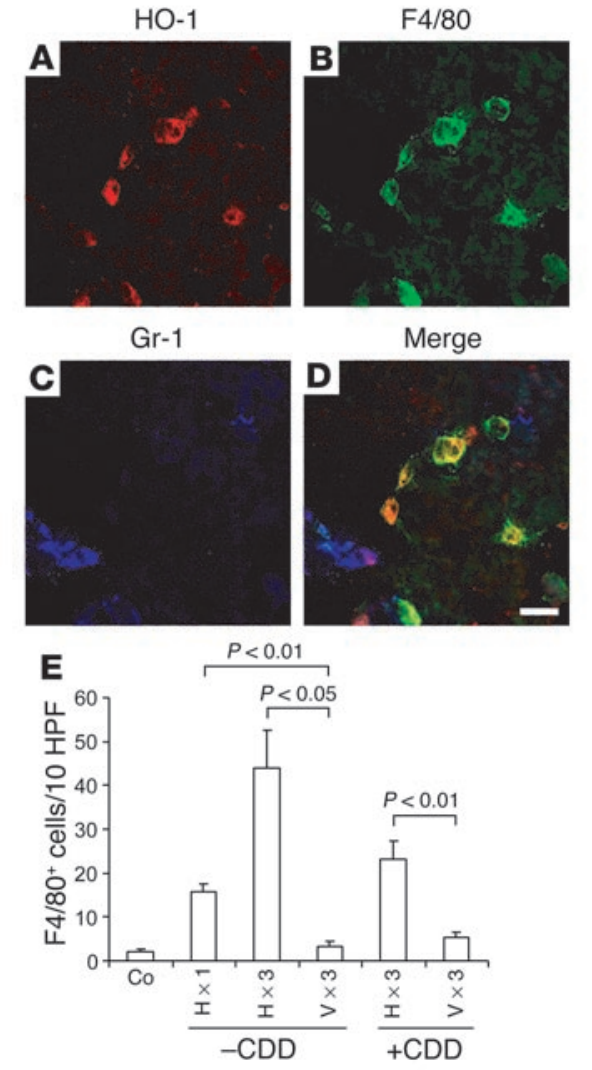

\section{Figure 3}

Hemin increases $\mathrm{HO}-1^{+}$pancreatic macrophages in association with MCP-1 and MIP-1 $\alpha$ induction. (A-D) Pancreas from CDD-fed mice was triple stained using antibodies to $\mathrm{HO}^{-1}$ (A), the macrophage marker F4/80 (B), and the granulocyte marker Gr-1 (C). (D) A merged image of the triple stain. Scale bar: $20 \mu \mathrm{m}$. (E) Mice were treated i.p. with hemin or vehicle 1 or 3 times during a 1-week period followed by feeding with CDD or regular chow for 3 days. Pancreata were collected followed by staining and counting F4/80+ macrophages in 10 randomly selected high-power fields (HPFs). The mean \pm SD (3 mice/group) and $P$ values for the indicated comparisons are shown. (F) Hemin or vehicle was injected i.p. once followed by harvesting of pancreata after 24 hours. A noninjected control group was also included. mRNA levels were then estimated from pancreata of 3 mice per group as a ratio of the indicated chemokine to $\beta$-actin (mean \pm SD). Changes in RANTES/MIP-2 mRNA levels were not significant after vehicle or hemin administration.

$(P<0.05)$ (Figure 5C). This was further confirmed by histological assessment of the pancreata (Figure 5, B, D, and E). These results indicate that the observed effect of hemin is not directly related to hemin but rather to hemin-activated macrophages that home to the pancreas and exert their protective effect.

In order to determine whether the protective effect of hemin is mediated via HO-1, we used small interfering RNA (siRNA) to blunt the hemin-induced upregulation of HO-1. First, we tested 2 siRNA constructs for their ability to inhibit HO-1 induction of the peritoneal cells that are used for cell transfer. siRNA construct 2 had a dramatic effect in blocking HO-1 induction (Figure 6, A and B). Construct 2 and the scrambled construct were then used to pretreat mice (together with hemin on alternate days) followed by transfer to naive mice, then initiation of CDD feeding (see Figure 6C or experimental design). Mice that were recipients of peritoneal cells treated with HO-1 siRNA/ hemin had a significantly worse histology score as compared with mice that received peritoneal cells treated with scrambled siRNA/hemin (Figure 6D). This was also supported by immunofluorescence staining of the pancreata, which showed that mice receiving scrambled-pretreated peritoneal cells had significant pancreatic costaining of the $\mathrm{F} 4 / 80^{+}$macrophages with $\mathrm{HO}-1$ in contrast with the limited pancreatic $\mathrm{HO}-1^{+}$staining in mice that received peritoneal cells pretreated with HO-1 siRNA (Figure 6, E-J). Collectively, these results indicate that the protective effects of hemin are mediated via $\mathrm{HO}-1$ and hemin-activated $\mathrm{HO}-1^{+}$ macrophages that home to the pancreas.

\section{Discussion}

Acute pancreatitis can be a severely debilitating if not lethal disease in humans. Most therapies are supportive and target the hemodynamic effects, such as dehydration, by removal of precipitating factors that include alcohol and biliary obstructing calculi $(25,26)$. While the findings of this study deal with mouse experimental pancreatitis, they do offer a novel approach of immune-mediated therapy via the unanticipated effect of hemin on leukocyte recruitment to the pancreas that may have potential relevant human applications. In our experimental system, we carried out 2 types of cell transfers. For both types of transfer experiments, the hemin-primed macrophages were derived from donor mice that were treated with 3 doses of hemin (excessive dosing decreased the level of HO-1 induction; Figure 1C). The difference between the bioluminescence transfer study (Figure 4) and the other transfer experiments that involved CDD feeding (Figures 5 and 6 ) is that in the latter CDD-related experiments hemin priming of the recipient mice is not required due to the effect of CDD on the pancreas, which triggers homing of the transferred cells to the pancreas. In contrast, for the bioluminescence homing experiment (Figure 4), a single dose of hemin priming was necessary since macrophages were not recruited to the pancreas unless the recipients received the hemin priming, which stimulates pancreatic chemokine production and triggers migration of macrophages to the pancreas (Figure 3). Notably, administration of panhematin (which is used clinically in patients; ref. 13-16) into mice i.p. induced high pancreatic HO-1 levels as seen with hemin (not shown). Although the relevant in vivo chemokines that are involved in macrophage recruitment to the pancreas remain to be defined, a cellular therapeutic approach may also be envisioned whereby autologous monocytes can be activated by hematin ex vivo followed by reinfusion.

The demonstrated benefit of hemin or hemin-activated cell therapy in experimental mouse pancreatitis is prophylactic and if translated to potential human use may be relevant to high-risk patients undergoing endoscopic retrograde cholangiopancreatograms who may develop pancreatitis (27). However, additional studies in experimental systems would be needed to test the prophylactic and therapeutic effects of different routes of hemin or cell therapy administration. To that end, treatment of mice with hemin (as in Figure 1) or hemin-primed peritoneal cells (as in Figure 5) 1.5 days after starting CDD did not afford any significant protection from pancreatic injury (data not shown). Further stud- 
A

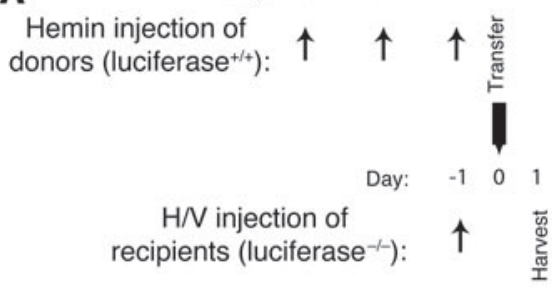

$5 \mathrm{~min}$
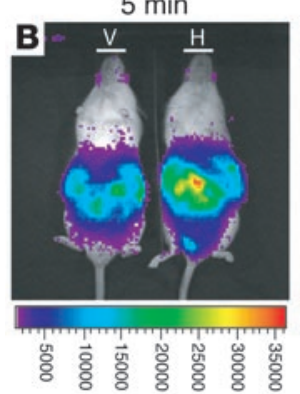

Luciferase

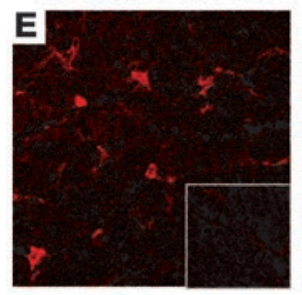

$24 \mathrm{~h}$

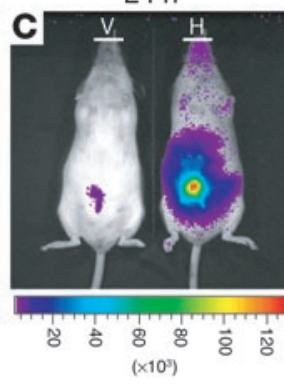

$\mathrm{F} 4 / 80$

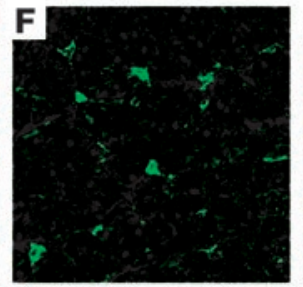

$24 \mathrm{~h}$
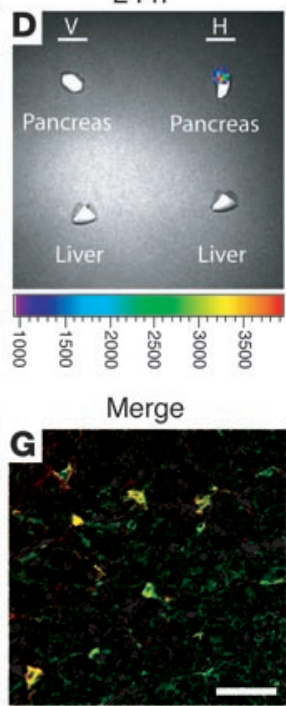

ies will be necessary to determine whether hemin-based therapy can also be protective at specific windows of opportunity after onset of pancreatitis or if the benefit is strictly prophylactic.

The observed effects of hemin are related to HO- 1 activation, which can unveil several potential protective mechanisms consequent to 1 or more of the HO-1 downstream byproducts

\section{Figure 4}

Effect of hemin on in vivo macrophage homing to the pancreas using bioluminescence imaging. (A) Experimental scheme of cell transfer. Hemin was injected 3 times i.p. (arrows) into luciferaseoverexpressing (luciferase ${ }^{+/+}$) mice, then peritoneal cells were harvested and macrophages were selected using anti-Mac-1 magnetic beads. Luciferase/Mac-1 double-positive cells were transferred i.p. to wild-type (luciferase ${ }^{-/}$) mice that were preinjected with 1 dose of hemin or vehicle 24 hours prior to the transfer. This hemin injection was necessary to induce pancreatic chemokines (Figure 3F). (B-D) Live images of recipient intact anesthesized mice were taken 5 minutes (B) or 24 hours (C) after cell transfer. Livers and pancreata were removed 24 hours after the transfer, followed by imaging (D). The signal intensity scale bar is shown below each image. (E-G) A duplicate of the pancreata shown in part $\mathbf{D}$ (from recipients receiving donor cells from hemin-injected animals) was double stained with antibodies to luciferase $(E)$ and F4/80 (F). Similar double staining of pancreata from recipient animals receiving donor cells from V-injected mice showed background staining (e.g., E [inset] for the anti-luciferase staining). (G) A merged image of the double stain. Scale bar: $50 \mu \mathrm{m}$.

carbon monoxide, biliverdin, or ferritin (4-6). For example, $\mathrm{HO}-1$-drived carbon monoxide has antiinflammatory effects mediated by IL-10 signaling and p38 mitogen-activated protein kinase activity $(3,28)$. The effects of the 3 major HO-1 by-products on pancreatitis and its progression remain to be investigated. Our findings suggest a model whereby hemin upregulates several pancreatic chemokines (e.g., MCP-1 and MIP- $1 \alpha$, with the relevant in vivo chemokines remaining to be defined) and causes a significant increase in $\mathrm{HO}-1^{+}$ peritoneal macrophages and other cells (Figure 7). Chemokine induction, in turn, triggers the recruitment of $\mathrm{HO}-1^{+}$peritoneal macrophages to the pancreas. Our data also demonstrate that the peritoneal activated cells and not a direct hemin effect are responsible for imparting the observed protection from pancreatitis-related injury.
A Day: $\begin{array}{lllllllll}-5 & -4 & -3 & -2 & -1 & 0 & 1\end{array}$

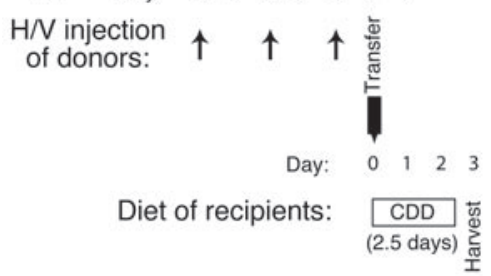

C

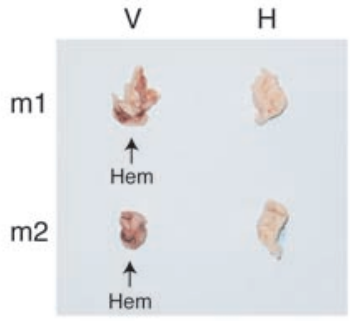

B

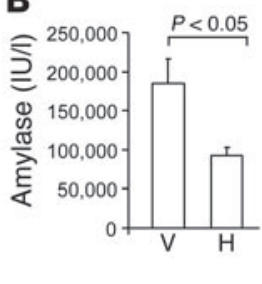

$\mathrm{V}$
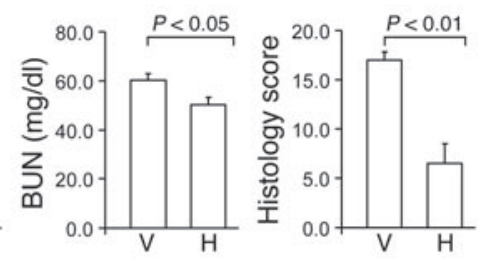

$\mathrm{H}$
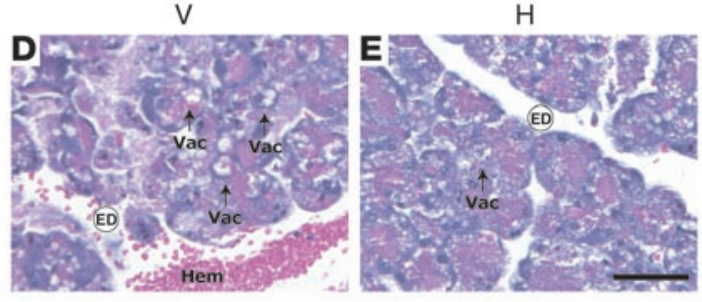

\section{Figure 5}

Protective effect of hemin-primed peritoneal cells on CDD-induced pancreatitis. (A) Peritoneal cells are isolated from hemin- or vehicle-pretreated mice (3 injections indicated by arrows), then transferred i.p. into naive recipient littermates followed by immediate initiation of CDD feeding. (B-E) After 2.5 days of CDD, sera were collected to measure amylase and BUN (mean \pm SD, 6 mice/group). Pancreata were also collected for gross tissue assessment and for histological staining and scoring. Scale bar: $50 \mu \mathrm{m}$. Vac, vacuoles; ED, edema; Hem, hemorrhage; m1 and m2, pancreatic tissues from 2 separate mice per hemin- or vehicle-pretreated group. 


\begin{tabular}{|c|c|c|c|c|c|c|}
\hline Day: & 1 & 2 & 3 & 4 & 5 & 6 \\
\hline RNA (i.p.) & $\uparrow$ & & $\uparrow$ & & $\uparrow$ & \\
\hline
\end{tabular}

B

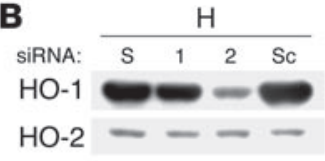

C

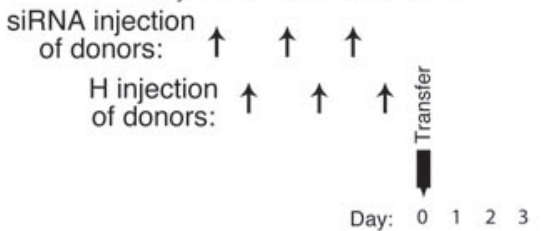

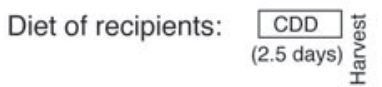
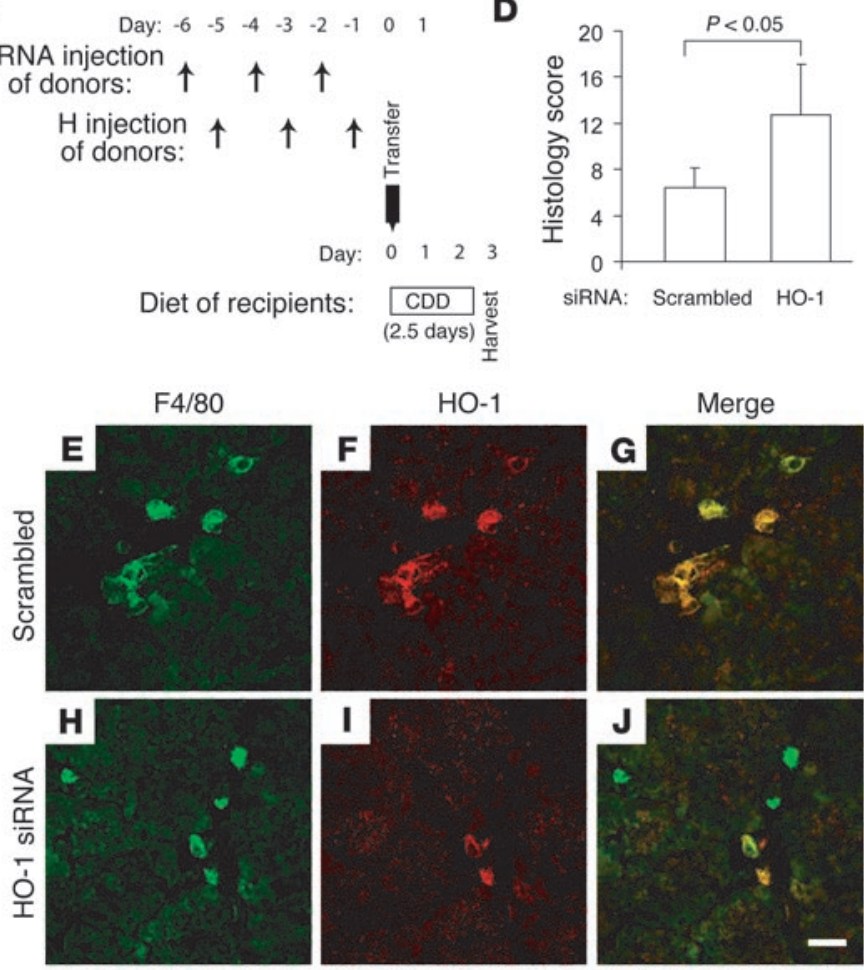

\section{Methods}

Hemin, vehicle, and antibodies. Hemin (Sigma-Aldrich) was dissolved in 10\% ammonium hydroxide in $0.15 \mathrm{M} \mathrm{NaCl}$ to prepare a stock solution of 100 $\mathrm{mg} / \mathrm{ml}$, then further diluted 1:40 with sterile $0.15 \mathrm{M} \mathrm{NaCl}$ and injected into mice $(10 \mu \mathrm{l} / \mathrm{g})$. Vehicle-injected mice received an identical $\mathrm{NH}_{4} \mathrm{OH}$-containing solution lacking hemin. The antibodies used were directed to the following: HO-1 and HO-2 (Stressgen Biotechnologies); vimentin, smooth muscle actin, and luciferase (NeoMarkers); allophycocyanin-conjugated Gr-1 and Mac-1 (BD Biosciences - Pharmingen); and FITC-conjugated F4/80 (Serotec). Panhematin was kindly provided by Stephen Collins (Ovation Pharmaceuticals, Inc., Deerfield, Illinois, USA).

Mice and pancreatitis models. All animal protocols were approved by the Institutional Animal Care and Use Committees of Stanford University and the VA Palo Alto Health Care System. BALB/c mice were housed under pathogen-free conditions and utilized in 2 established models of pancreatitis $(22-24,29,30)$. For caerulein-induced pancreatitis, age- and sex-matched mice were fasted for 12-16 hours but allowed water ad libitum. Mice then received 7 hourly i.p. injections of saline (control group) or $50 \mu \mathrm{g} / \mathrm{kg}$ caerulein (Research Plus) in saline and were followed up to 12

\section{Figure 7}

Schematic of the effect of hemin on mouse $\mathrm{HO}-1$ induction and homing of peritoneal macrophages to the pancreas. Hemin induced the expression of several pancreas chemokines and increased peritoneal macrophages and their HO-1 expression. Peritoneal macrophages that overexpress $\mathrm{HO}-1$ homed to the pancreas and were able to provide significant protection from pancreatic injury.

\section{Figure 6}

Inhibition of the protective effect of hemin-primed peritoneal cells using HO-1 knock-down in vivo. (A and $\mathbf{B}$ ) Peritoneal cells were isolated from hemin-treated mice that were also injected i.p. (arrows) on alternate days with saline, HO-1 siRNA (constructs 1 and 2), or scramble control (Sc) siRNA. The peritoneal cells were then used to examine $\mathrm{HO}-1$ and $\mathrm{HO}-2$ levels by immunoblotting. (C and D) Peritoneal cells were harvested from hemin/construct 2 HO-1 siRNA or hemin/scrambled siRNA-injected mice, then transferred into naive recipient littermates followed by immediate initiation of CDD feeding. At 2.5 days of CDD feeding, pancreata were collected for histologic scoring. Data for histology score are presented as mean $\pm \mathrm{SD}(n=5-6 /$ group $)$, and are $6.4 \pm 1.7$ (scrambled) and $12.7 \pm 4.5$ (HO-1 siRNA). (E-J) Pancreata from C and $\mathbf{D}$ were double stained using antibodies to F4/80 and HO-1. Scale bar, $20 \mu \mathrm{m}$.

hours. For the pancreatitis induced by feeding a choline-deficient diet (CDD), young female mice (15-19 g) were fasted, then fed CDD (Harlan Teklad) supplemented with 0.5\% DL-ethionine (SigmaAldrich) or normal chow (control group) for 3 days, then switched to a normal diet for 1, 2, or 5 days.

Animal procedures. Mice were euthanized by $\mathrm{CO}_{2}$ inhalation, then pancreata and lungs were rapidly removed, divided into 3-4 pieces, and blood was collected by intracardiac puncture. Individual lung and pancreas fragments were immediately fixed in $10 \%$ formalin, embedded in Optimum Cutting Temperature medium (Sakura Finetek Inc.) or snap-frozen in liquid $\mathrm{N}_{2}$ for subsequent protein, enzyme, and RNA analysis $(29,30)$. Fixed tissues were sectioned then stained using H\&E (performed by Histo-Tec Laboratory). Immunofluorescence staining was performed as described (29), and images were analyzed using confocal microscopy. Total tissue lysates were prepared by homogenization in Laemmli sample buffer and analyzed by SDS-PAGE followed by transfer to membranes for blotting and protein visualization by enhanced chemiluminescence. Trypsin activity was determined using a fluorimetric assay and the substrate Boc-Gln-Ala-Arg-MCA (Peptides International) as well as a comparison to a standard curve generated with purified trypsin as described (31). For MPO activity, lung and pancreas tissues were processed as described (32) followed by the use of an

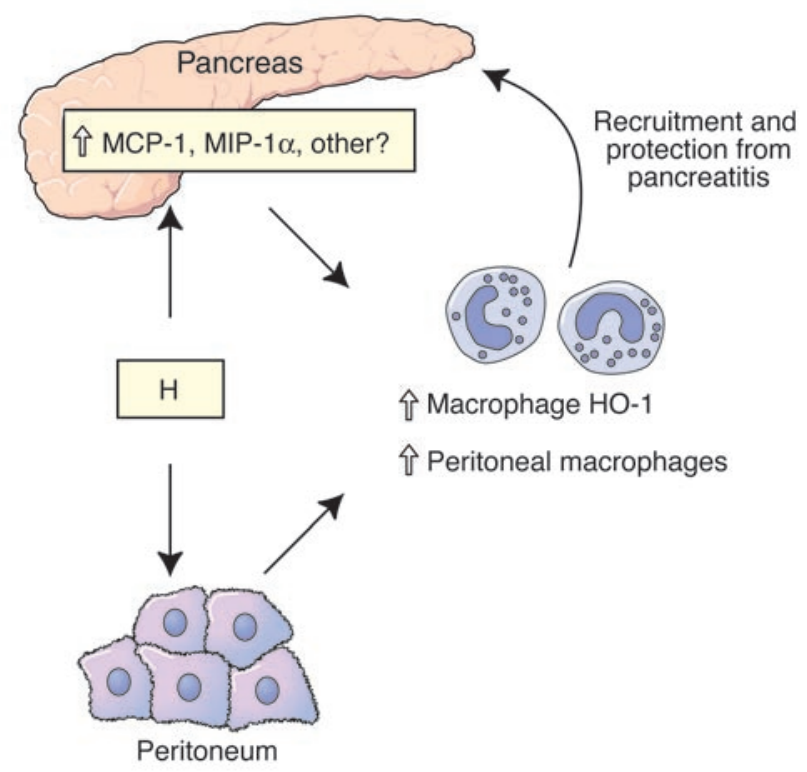


MPO kit according to manufacturer guidelines (Calbiochem). Lung injury after caerulein administration was assessed as previously described based on neutrophilic infiltration, pulmonary edema, alveolar distension, and collapse (32). $P$ values were calculated for histology, enzyme, and serum test comparisons using the paired 1-tailed Student's $t$ test and for survival comparisons using the $\chi^{2}$ method.

Real-time RT-PCR. Total RNA was isolated from pancreatic tissue using a commercial kit (Tel-Test Inc.). First-strand cDNA was synthesized using oligo-dT primers and SuperScript II reverse transcriptase (Invitrogen Corp.). Real-time quantitative PCR was performed with an ABI Prism 7900 Sequence Detection System (Applied Biosystems) and established primers for MCP-1, MIP-1 $\alpha$, MIP-2, RANTES, and $\beta$-actin $(30,33,34)$. Primers for $\beta$-actin were used as an internal control reference, and the amount of specific cytokine relative to actin transcript was determined and reported as mean \pm SD.

Luciferase transgenic mice and imaging. The luciferase-overexpressing transgenic mice (in an $\mathrm{FVB} / \mathrm{n}$ background) and their nontransgenic counterparts (35) received 3 doses of hemin on days $-5,-3$, and -1 . On day 0 , peritoneal cells were harvested, and the Mac- $1^{+}$cells were selected using Mac-1-coated (anti-CD11b) microbeads (Miltenyi Biotec). Luciferase/Mac-1 double-positive cells $\left(2 \times 10^{6}\right)$ were transferred i.p. to wild-type FVB/n mice pretreated a day earlier with 1 dose of hemin or vehicle in order to prime cellular homing. Bioluminescent in vivo images of the recipient mice were obtained 5 minutes and 24 hours after cell transfer as described (35). Mice that were imaged 24 hours after transfer were then killed followed by isolation of several organs for luciferase imaging and immunofluorescence staining.

Hemin-pretreated peritoneal cell transfer into CDD-fed mice. Peritoneal cells were harvested from hemin- or vehicle-pretreated (3 doses given every other day) young female BALB/c mice (15-19 g). Isolated cells were washed with $\mathrm{PBS}$, and equivalent cells were transferred into naive $\mathrm{BALB} / \mathrm{c}$ littermates i.p. followed by initiation of CDD feeding. Mice were sacrificed after 2.5 days of CDD feeding, followed by isolation of the pancreata (for histological and fluorescence staining) and blood. Serum was isolated from clotted blood followed by measurement of amylase and BUN using standard methods. The severity of pancreatitis was scored using established histological criteria that assigned individual numerical scores (1, mild; 2 , moderate; and 3 , severe) to edema, inflammation, and hemorrhage and alternate scores for parenchymal necrosis (3, focal; 5, sublobular; and 7, lobular) (36).

HO-1 siRNA and hemin-pretreated cell transfer. Mice were injected i.p. every other day ( 3 total injections) with either saline or with 2 HO- 1 siRNA constructs ( $2 \mathrm{mg} / \mathrm{kg}$ body weight) or with a scrambled siRNA sequence. Hemin was injected in the intervening days (an additional 3 injections). After 7 days, peritoneal cells were harvested and analyzed by immunoblotting using antibodies to HO- $1 / 2$. Since significant inhibition of $\mathrm{HO}-1$ occurred with construct 2, construct 2 was used for subsequent transfer of peritoneal cells (pretreated with hemin and scrambled siRNA or with hemin and HO-1 siRNA) to naive recipient mice that were then fed CDD for 2.5 days followed by isolation of the pancreata. The siRNAs were synthesized by Dharmacon and reported previously (37). The sense and antisense strand sequences of the $2 \mathrm{HO}-1$ siRNA and nonspecific siRNA scrambled duplex were as follows: 1, 5'-UGGCUUCCUUGUACCAUAUdTdT-3' (sense) and 5'-AUAUGGUACAAGGAAGCCAdTdT-3' (antisense); 2, 5'-GCCACACAGCACUAUGUAAdTdT-3' (sense) and 5'-UUACAUAGUGCUGUGUGGCdTdT-3' (antisense); and scrambled, 5'-GCGCGCUUUGUAGGAUUCGdTdT3' (sense) and 5'-CGAAUCCUACAAAGCGCGCdTdT-3' (antisense).

\section{Acknowledgments}

This work was supported by Department of Veterans Affairs Merit Awards (E.C. Butcher and M.B. Omary), NIH grant DK52951 (M.B. Omary), and NIH Digestive Disease Center Grant DK56339. A. Habtezion is supported by an NIH Training Grant DK07056 postdoctoral fellowship. We are grateful to Stephen Collins (Ovation Pharmaceuticals Inc.) for providing us with panhematin; Evelyn Resurreccion for assistance with tissue sectioning and immunofluorescence staining; Tohru Sato for helpful discussions; Tim Doyle for assistance with bioluminescence imaging; and Diana Toivola and Kris Morrow for assistance with figure preparation.

Received for publication March 2, 2005, and accepted in revised form August 23, 2005.

Address correspondence to: Bishr Omary, 3801 Miranda Avenue, Mail code 154J, Palo Alto, California 94304, USA. Fax: (650) 8523259; E-mail: mbishr@stanford.edu.

Ikuo Nakamichi and Aida Habtezion contributed equally to this work.

Ikuo Nakamichi's present address is: Kyushu University Hospital, Fukuoka, Japan.
1. Poss, K.D., and Tonegawa, S. 1997. Heme oxygenase 1 is required for mammalian iron reutilization. Proc. Natl. Acad. Sci. U. S. A. 94:10919-10924.

2. Soares, M.P., et al. 1998. Expression of heme oxygenase- 1 can determine cardiac xenograft survival. Nat. Med. 4:1073-1077.

3. Lee, T.S., and Chau, L.Y. 2002. Heme oxygenase-1 mediates the anti-inflammatory effect of interleukin-10 in mice. Nat. Med. 8:240-246.

4. Otterbein, L.E., Soares, M.P., Yamashita, K., and Bach, F.H. 2003. Heme oxygenase-1: unleashing the protective properties of heme. Trends Immunol. 24:449-455.

5. Durante, W. 2003. Heme oxygenase-1 in growth control and its clinical application to vascular disease. J. Cell. Physiol. 195:373-382.

6. Sikorski, E.M., Hock, T., Hill-Kapturczak, N., and Agarwal, A. 2004. The story so far: molecular regulation of the heme oxygenase- 1 gene in renal injury. Am. J. Physiol. Renal Physiol. 286:F425-F441.

7. Tenhunen, R., Marver, H.S., and Schmid, R. 1968. The enzymatic conversion of heme to bilirubin by microsomal heme oxygenase. Proc. Natl. Acad. Sci. U. S. A. 61:748-755.

8. Fu, K., Sarras, M.P., Jr., De Lisle, R.C., and Andrews,
G.K. 1997. Expression of oxidative stress-responsive genes and cytokine genes during caerulein-induced acute pancreatitis. Am. J. Physiol. 273:G696-G705.

9. Sato, H., et al. 1997. Expression of stress proteins heme oxygenase- 1 and -2 in acute pancreatitis and pancreatic islet betaTC3 and acinar AR42J cells. FEBS Lett. 405:219-223.

10. Yet, S.F., et al. 1999. Hypoxia induces severe right ventricular dilatation and infarction in heme oxygenase-1 null mice. J. Clin. Invest. 103:23-29.

11. Yachie, A., et al. 1999. Oxidative stress causes enhanced endothelial cell injury in human heme oxygenase-1 deficiency. J. Clin. Invest. 103:129-135.

12. Kawashima, A., Oda, Y., Yachie, A., Koizumi, S., and Nakanishi, I. 2002. Heme oxygenase-1 deficiency: the first autopsy case. Hum. Pathol. 33:125-130.

13. Tenhunen, R., and Mustajoki, P. 1998. Acute porphyria: treatment with heme. Semin. Liver Dis. 18:53-55.

14. Dellon, E.S., et al. 2002. Treatment of recurrent allograft dysfunction with intravenous hematin after liver transplantation for erythropoietic protoporphyria. Transplantation. 73:911-915.

15. Rund, D., and Rachmilewitz, E. 2000. New trends in the treatment of beta-thalassemia. Crit. Rev.
Oncol. Hematol. 33:105-118.

16. Anderson, K.E., et al. 2005. Recommendations for the diagnosis and treatment of the acute porphyries. Ann. Intern. Med. 142:439-450.

17. Ishikawa, K., et al. 2001 . Heme oxygenase- 1 inhibits atherosclerotic lesion formation in ldl-receptor knockout mice. Circ. Res. 88:506-512.

18. Kanakiriya, S.K., et al. 2003. Heme: a novel inducer of MCP-1 through HO-dependent and HO-independent mechanisms. Am. J. Physiol. Renal Physiol. 284:F546-F554.

19. Alam, J., et al. 2003. Heme activates the heme oxygenase-1 gene in renal epithelial cells by stabilizing Nrf2. Am. J. Physiol. Renal Physiol. 284:F743-F752.

20. Graca-Souza, A.V., Arruda, M.A., de Freitas, M.S., Barja-Fidalgo, C., and Oliveira, P.L. 2002. Neutrophil activation by heme: implications for inflammatory processes. Blood. 99:4160-4165.

21. Gukovskaya, A.S., et al. 2002. Neutrophils and NADPH oxidase mediate intrapancreatic trypsin activation in murine experimental acute pancreatitis. Gastroenterology. 122:974-984.

22. Jensen, R.T., Lemp, G.F., and Gardner, J.D. 1980. Interaction of cholecystokinin with specific membrane receptors on pancreatic acinar cells. Proc. 
Natl. Acad. Sci. U. S. A. 77:2079-2083.

23. Lombardi, B., Estes, L.W., and Longnecker, D.S 1975. Acute hemorrhagic pancreatitis (massive necrosis) with fat necrosis induced in mice by DLethionine fed with a choline-deficient diet. Am. J. Pathol. 79:465-480.

24. Algul, H., et al. 2002. Acute experimental pancreatitis and NF-kappaB/Rel activation. Pancreatology. 2:503-509.

25. Banks, P.A. 1997. Practice guidelines in acute pancreatitis. Am. J. Gastroenterol. 92:377-386.

26. Baron, T.H., and Morgan, D.E. 1999. Acute necrotizing pancreatitis. N. Engl. J. Med. 340:1412-1417.

27. Maschi, E., Mariani, A., Curioni, S., and Testoni, P.A. 2003. Risk factors for pancreatitis following endoscopic retrograde cholangiopancreatography: a meta-analysis. Endoscopy. 35:830-834.

28. Otterbein, L.E., et al. 2000. Carbon monoxide has anti-inflammatory effects involving the mito- gen-activated protein kinase pathway. Nat. Med. 6:422-428.

29. Toivola, D.M., Baribault, H., Magin, T., Michie, S.A., and Omary, M.B. 2000. Simple epithelial keratins are dispensable for cytoprotection in two pancreatitis models. Am. J. Physiol. Gastrointest. Liver Physiol. 279:G1343-G1354.

30. Zhong, B., et al. 2004. Organ-specific stress induces mouse pancreatic keratin overexpression in association with NF-kappaB activation. J. Cell Sci. 117:1709-1719.

31. Nathan, J.D., et al. 2005. Transgenic expression of pancreatic secretory trypsin inhibitor-I ameliorates secretagogue-induced pancreatitis in mice. Gastroenterology. 128:717-727.

32. Oruc, N., et al. 2004. Infliximab: a new therapeutic agent in acute pancreatitis? Pancreas. 28:e1-e8.

33. Zhang, Y., McCormick, L.L., Desai, S.R., Wu, C., and Gilliam, A.C. 2002. Murine sclerodermatous graft- versus-host disease, a model for human scleroderma: cutaneous cytokines, chemokines, and immune cell activation. J. Immunol. 168:3088-3098.

34. Walzog, B., et al. 1999. A role for beta(2) integrins $(\mathrm{CD} 11 / \mathrm{CD} 18)$ in the regulation of cytokine gene expression of polymorphonuclear neutrophils during the inflammatory response. FASEB J. 13:1855-1865.

35. Cao, Y.A., et al. 2004. Shifting foci of hematopoiesis during reconstitution from single stem cells. Proc. Natl. Acad. Sci. U. S. A. 101:221-226.

36. Spormann, H., Sokolowski, A., and Letko, G. 1989. Effect of temporary ischemia upon development and histological patterns of acute pancreatitis in the rat. Pathol. Res. Pract. 184:507-513.

37. Zhang, X., et al. 2004. Small interfering RNA targeting heme oxygenase- 1 enhances ischemiareperfusion-induced lung apoptosis. J. Biol. Chem. 279:10677-10684. 